\title{
THEORETICAL MODELS OF W VIRGINIS VARIABLES
}

\author{
Alan Bridger \\ Royal Greenwich Observatory, Herstmonceux Castle, Ha1lsham, \\ East Sussex, BN27 1RP, England.
}

\section{INTRODUCTION}

W Virginis variables are the population II counterparts of the classical cepheids, although they do not show quite the same trends as are seen in the latter. Theoretical studies of the population II cepheids have not been very extensive until recent studies of the shorter period variables (BL Herculis variables, with periods between 1 and 10 days). The variables with periods above 10 days (up to about 50 days) have only been studied by a few authors, modelling the prototype star (W Vir) (.g. Christy 1966; Davis 1974). Although these models qualitatively reproduced the observations they were not very successful, and were based on a stellar mass $\left(0.88 \mathrm{M}_{Q}\right)$ that now seems likely to be too high.

This paper summarises the results of a series of hydrodynamic (nonlinear) models constructed in an attempt to reproduce the observed characteristics of these stars. The full results and analysis are given elsewhere (Bridger 1984). Conventional non-linear methods are used for the modelling, including attempts to improve on previously used outer dynamic boundary conditions. Convection is ignored. A realistic equation of state is used, with opacities calculated by Carson (1976) for a composition of $\mathrm{Y}=0.25, \mathrm{Z}=0.005$.

OBSERVATIONAL DATA

Observations of W Virginis variables are unfortunately rather scarce, This is especially the case for velocity observations. Very few observed stars have well defined velocity curves, only $W$ Virginis itself being well observed. The major thing that the velocity observations do tell us is that the stars with periods above 13 days have highly asymmetric velocity curves frequently accompanied by hydrogen emission lines at rise to maximum light. This is probably caused by strong outward moving shock waves.

Kwee (1967) gives some good light curves of some field W Vir stars, and splits the curves into two classes which he calls "crested" (C-type) and "flat-topped" (F-type). The stars with periods less than 13 days usually fit neither class, having rather featureless light curves ( $X-$ type). 
A study has been made of the best available observations, producing new estimates of luminosity $\left(\log \left(L / L_{\theta}\right)\right)$ and effective temperature $\left(\log \mathrm{T}_{e}\right)$ for the stars. The study shows that the $\mathrm{C}$ - and $\mathrm{F}$-type variables (hereinafter the variables are typed according to their light curve) occupy different positions in the HR diagram. Following Kwee two almost parallel Period-Radius ( $P-R$ ) relations can be derived for the two classes. Figure 1 is an HR diagram showing the positions of the variables, observed blue and red edges (Demers \& Harris 1974), a theoretical blue edge based on the Carson opacties (Worre11, private communication) and a red edge estimated from static models.

This division may turn out to be due to different evolutionary tracks. The mechanism for feeding stars into the population II instability strip at this point seems to be "blue-looping" from the Asymptotic Giant Branch (AGB) caused by thermal instabilities in the helium burning shell (e.g. Mengel 1973). Making simple assumptions (that metallicity varies little or that it has little effect on the evolution), then it may be that a slightly higher mass star moving up the AGB executes this blue-1oop at a later stage, causing the star to have a higher luminosity when passing through the instability strip, producling a C-type variable. Thus we see that the C-type variables may be slightly more massive than the F-type. Assuming the two classes follow paralle1 $P-R$ relations then the mass ratio may be estimated, giving $\mathrm{M}_{C} \sim 1.3 \mathrm{M}_{F}$. This fits nicely into the range of possible masses given by Bohm-Vitense et a1 (1974) of $0.5<M / M_{\Theta}<0.75$.

\section{SURVEY RESULTS}

The survey consisted of a series of 25 models constructed to cover the Instability Strip, aiming at periods in the range $10-20$ days. A mass of $0.6 \mathrm{M}_{\odot}$ was used, with a few additional models constructed with masses of $0.5 \mathrm{M}_{\Theta}$ and $0.8 \mathrm{M}_{\Theta}$. The survey models were then looked at in the same way as the observational data.

Figure 1. HR diagram

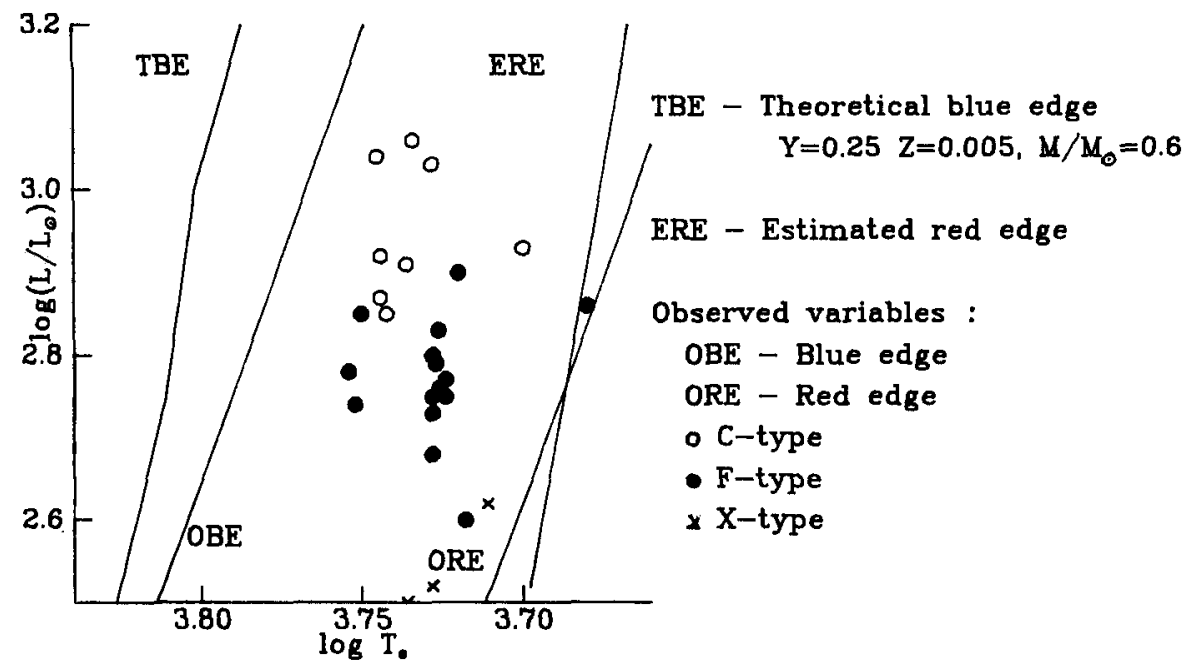


The modelled light curves also show the split into $\mathrm{C}$ - and F-type curves, at the approximate expected stellar parameters, with the derived $P-R$ relation agreeing well with the observed relations, to within estimated observational errors. The general agreement with the observed curves is good, although it did prove more difficult to reproduce the $\mathrm{F}$-type curves (figure 2 ). Using a mass of $0.5 \mathrm{M}_{\Theta}$ seemed to make little difference to the general pulsational characteristics (except, of course, to the period), but using $0.8 \mathrm{M}_{\Theta}$ produced very different curves. It seemed likely that that the observed light curves could be reproduced using this mass and reasonable values of the stellar parameters. Thus $0.8 \mathrm{M}_{\Theta}$ seems to be too high to be the mass of most of these variables. This is quite reasonable as it is at the upper limit of the possible evolutionary masses in any case. The amplitudes of the velocity curves are quite reasonable, and the curves for many models show the high asymmetry and strong shocks that presumably accompany the hydrogen emssion lines seen in some stars.

A few models showed very slight "RV Tauri behaviour" in their light and/or velocity curves. This is the alternation of larger and smaller amplitudes, and possibly also longer and shorter periods, causing a doubling of the repetition period. In particular one model showed this behaviour to a large extent (figure 3), and also was capable of switching to a more normal variation and back again.

Further models were calculated using Los Alamos opacities. Two of these used the same composition as the survey, and the same parameters as two of the survey models. The light curves computed were not as close to those observed as the survey models were, being significantly different in shape and the position of the secondary bump. A model using $Y=$ 0.299 (the King Ia mix, Cox \& Tabor 1976) showed a light curve

Figure 2. Examples of modelled curves

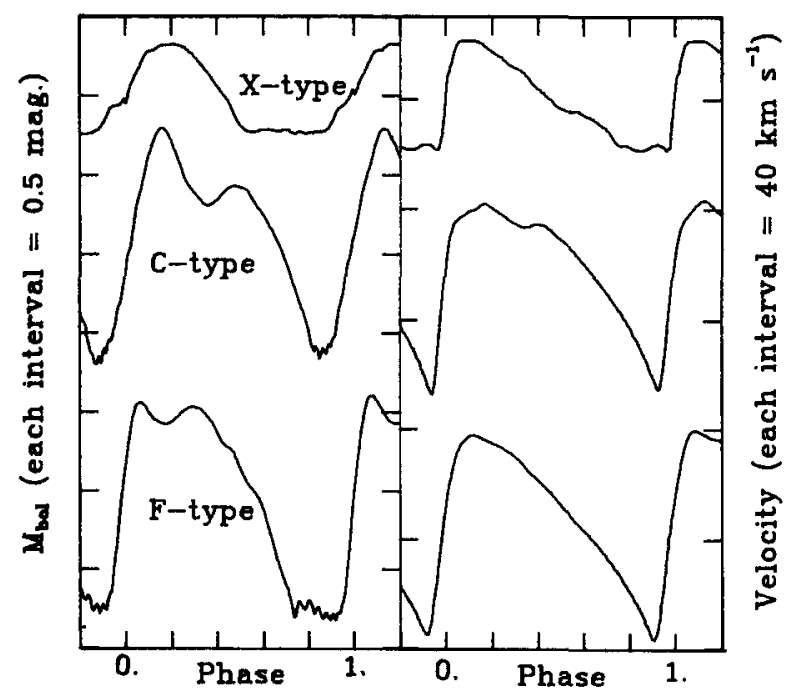

Figure 3. RV Tauri behaviour

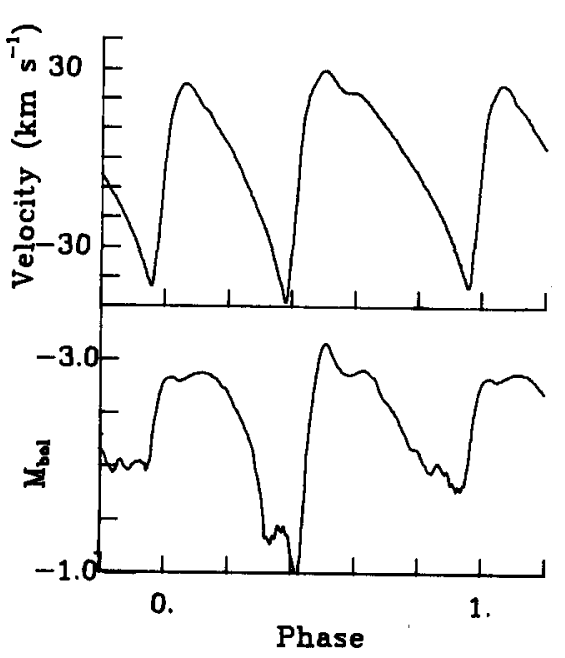


completely unlike those observed, suggesting that $30 \%$ Helium is too high for these stars.

Finally, three of the survey models proved to be very good models of individual stars, even without attempting to tune the parameters to improve the fit. One of these reproduces the light curve and general properties of the variable CS Cas very well. Table 1 shows the parameters of model and star and figure 4 compares their light curves.

\section{CONCLUSION}

This study shows that the W Virginis variables can be modelled using suitable input physics and stellar parameters derived from evolution or observation. Indeed the modelling can be very good. However it also shows that convection is probably very important for the cooler stars.

\section{REFERENCES}

Bohm-Vitense, E., Szkody, P., Wallerstein, G., and Iben, I., (1974). Astrophys. J., 194, 25.

Bridger, A. (1984). Ph. D. Thesis, University of St. Andrews.

Carson, T.R. (1976). Ann. Rev. Astron. \& Astrophys., 14, 95.

Christy, R.F., (1966). Astrophys. J., 145, 337.

Cox, A.N., \& Tabor, J.E., (1976). Astrophys. J. Supp1., 31, 271.

Davis, C.G., (1974). Astrophys. J., 187, 175.

Demers, S. \& Harris, W.E., (1974). Astron. J., 79, 627.

Kwee, K.K., (1967). Bull. Astron. Netherlands, 19, 260.

Menge1, J.G., (1973). In Variable Stars in Globular Clusters and related systems, ed. J.D. Fernie. IAU Col1. no. 21. Reidel.

Table 1. Comparison of CS Cas and model star.

$\phi_{\mathrm{B}} \quad$ Phase of secondary bump

$\phi_{\mathrm{B}}-\phi_{\mathrm{M}}$ Phase of bump from maximum

Parameter

$\log \left(\mathrm{L} / \mathrm{L}_{0}\right)$

$\log \mathrm{T}_{\mathrm{e}}$

Model CS Cas

Amplitude (mag.) $1.8 \quad 1.44$

Asymmetry in $\quad 3.2 \quad 2.0$

light curve

$\begin{array}{lll}\phi_{\mathrm{B}} & 0.40 & 0.48 \\ \phi_{\mathrm{B}}-\phi_{\mathrm{M}} & 0.27 & 0.25\end{array}$

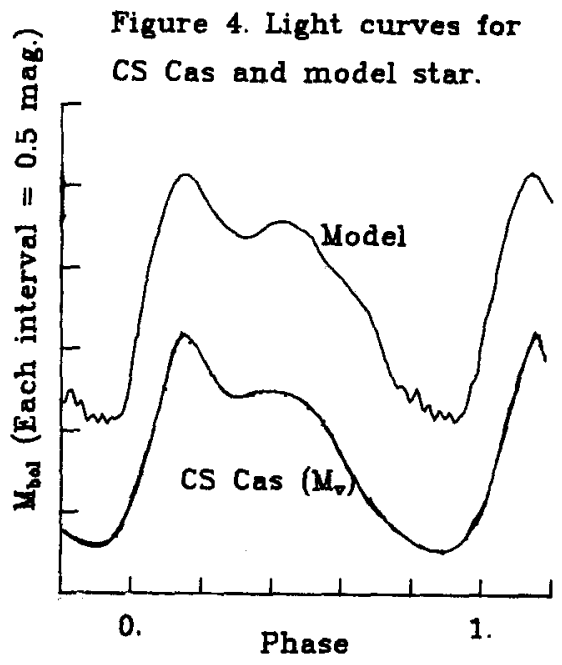

